\title{
Emergence in Biopharmaceuticals: The Role of Gedunin and Nimbin from Azadirachta indica as a potential cure for SARS CoV-2 and other viral diseases
}

\author{
Charunayan Kamath. $R$ \\ Vellore Institute of Technology, Vellore, Tamil Nadu, India email: charunayan@ gmail.com \\ Archanna J \\ Vellore Institute of Technology, Vellore, Tamil Nadu, India email: archannaj@gmail.com
}

Abstract: The COVID-19 pandemic caused by Severe Acute Respiratory Syndrome Corona virus 2 -SARS CoV- 2 has affected over 170 million people worldwide out of which 3.5 million have lost their lives so far. There is no effective remedy for this highly contagious disease to date. This study examines the effect of Gedunin and Nimbin, one of the constituents of Neem leaves (Azadirachta indica), especially as a possible prophylactic element and a remedy for Covid-19 using computational methods. Other main constituents of the neem leaves such as Azadirachtin and Nimbidin may also work as an active antiviral. This study was initiated by the insights gained from the traditional method of controlling respiratory ailments. We propose 'Emergence' as the property that dictates the need to use Gedunin and Nimbin along with the other bioactive components of neem leaves as adjuvants against coronaviruses and other viral diseases

Keywords: SARS CoV-2, Emergence, Gedunin, Nimbin, Neem, Coronavirus, Antiviral

"The whole is greater than the sum of the parts" - Aristotle

\section{Introduction}

Coronavirus disease 2019 (COVID-19) is an infectious disease caused by Severe Acute Respiratory Syndrome coronavirus 2 (SARSCoV-2). It was first identified in December 2019 in Wuhan, China, and has caused a pandemic (Chen et al., 2020). Coronaviruses are a family of viruses that can cause illnesses such as the common cold, Severe Acute Respiratory Syndrome (SARS) and the Middle East respiratory syndrome (MERS). People with COVID-19 have had a wide range of symptoms reported-ranging from mild symptoms to severe illness. The main symptoms reported are fever or chills, cough, shortness of breath or difficulty breathing, fatigue, muscle or body aches, headache, loss of taste or smell, sore throat, congestion or runny nose, nausea or vomiting and diarrhea (Grant et al., 2020). There are no specific antiviral treatments for COVID-19. Disease management involves the treatment 
of symptoms, supportive care, isolation, and experimental measures.

Coronaviruses are enveloped, positive singlestranded large RNA viruses that infect humans, but also a wide range of animals. Coronaviruses were first described in 1966 by Tyrell and Bynoe, who cultivated the viruses from patients with common colds (Grant et al., 2020; Velavan et al.,2020). Based on their morphology as spherical virions with a core-shell and surface projections resembling a solar corona, they were termed coronaviruses. Four subfamilies, namely alpha-, beta-, gamma- and delta-coronaviruses exist. While alpha- and beta-coronaviruses apparently originated from mammals, in particular from bats, gamma and delta-viruses originate from pigs and birds. The genome size varies between $26 \mathrm{~kb}$ and $32 \mathrm{~kb}$. Among the seven subtypes of coronaviruses that can infect humans, the beta-coronaviruses may cause severe disease and fatalities, whereas alphacoronaviruses cause asymptomatic or mildly symptomatic infections. SARS-CoV-2 belongs to the $\mathrm{B}$ lineage of the betacoronaviruses and is closely related to the SARS-CoV-2 virus. The major four structural genes encode the nucleocapsid protein $(\mathrm{N})$, the spike protein $(\mathrm{S})$, a small membrane protein (SM) and the membrane glycoprotein (M) with an additional membrane glycoprotein (HE) occurring in the HCoV-OC43 and HKU1 betacoronavirus (Tyrrell \& Bynoe,1966). SARSCoV-2 is $96 \%$ identical at the whole-genome level to a bat coronavirus.

The initial clinical sign of the SARS-CoV-2related disease COVID-19 which allowed case detection was pneumonia. More recent reports also describe gastrointestinal symptoms and asymptomatic infections, especially among young children (Rottier, 2013). Observations so far suggest a mean incubation period of five days and a median incubation period of 3 days (range: 0-24 days) (Chan et al.,2020). Like other viruses, SARS-CoV-2 infects lung alveolar epithelial cells using receptor-mediated endocytosis via the angiotensin-converting enzyme II (ACE2) as an entry receptor (Tyrrell \& Bynoe,1966). Artificial intelligence predicts that drugs associated with AP2-associated protein kinase 1 (AAK1) disrupting these proteins may inhibit viral entry into the target cells (Richardson et al.,2019). Baricitinib, used in the treatment of rheumatoid arthritis, is an AAK1 and Janus kinase inhibitor and suggested for controlling viral replication (Richardson et al.,2019). Moreover, an in vitro and a clinical study indicate that remdesivir, an adenosine analogue that acts as a viral protein inhibitor, has improved the condition in a few patients (Holshue et al., 2019; Wang et al., 2020). Chloroquine, by increasing the endosomal $\mathrm{pH}$ required for virus-cell fusion, has the potential of blocking viral infection (Wang et al., 2020) and was shown to affect the activation of p38 mitogen-activated protein kinase (MAPK), which is involved in the replication of $\mathrm{HCoV}$ 229E.

\section{Mpro Structure}

Further to the study of the structural features of the coronavirus, one of the most wellstudied structures is the $\mathrm{M}^{\text {pro }}$, also known as 
$3 \mathrm{CL}^{\text {pro. }}$. This is the main protease crucial for the replication of the viruses in the host cell (Anand et al., 2003; Hilgenfeld, 2014). With a $96 \%$ sequence similarity to the SARSCoronavirus $\mathrm{M}^{\text {pro }}$, the RMSD between the structures was found to be $0.53 \AA$ for the alpha carbons (Tan et al., 2005). The protein as such is made up of antiparallel $\beta$-barrel structures containing the active binding site amongst the domain I and II, i.e., the residues 10 to 182 covering both domains. Between 198 and 303is the III $^{\text {rd }}$ domain, responsible for the dimerization of the protein and is composed of 5 alpha - helices (Zhang et al., 2020). This dimerization acts as the catalyst by helping the formation of the $\mathrm{S} 1$ substratebinding pocket (Anand et al. 2002). The first eluted $\mathrm{M}^{\text {pro }}$ structure from the Wuhan variant of the disease can be retrieved from the protein data bank from the ID: 6LU7 and the same has been used in this docking study.

\section{Mechanism:}

SARS-CoV-2 enters the human cell by the interaction of the viral spike glycoprotein (S) with the ACE-2 receptor using the S1 binding domain and fuses with the host cell membrane with its S2 domain (Yang et al., 2010). Endosomal transport of the virus is carried out in the host cell cytoplasm and the viral genomic RNA is released. This RNA undergoes translation using the host machinery and produces complex pp1a and pplab proteins. However, the viral RNA also replicates to form the genetic material during the packaging of the viral progeny. $\mathrm{M}^{\mathrm{pro}}$ is formed by autolytic cleavage from pp1a and pplab (Jin et al., 2020) and further aids these complex polyproteins by mediating the proteolytic cleavage at 11 conserved sites to produce NSPs or non-structural proteins (Mengist et al., 2020). These NSPs help in the formation of the viral progeny which is released from the host cell for the further infection.

The viral $\mathrm{M}^{\text {pro }}$, helps the viruses at the most crucial step of replication and transcription of the virus, responsible for the increase of viral load. The importance of the protein, in addition to its viral exclusivity and non homogeneity to human cells, makes this protein a prime therapeutic target. This would also mean that one could expect minimal to no side effects when administered to humans. 


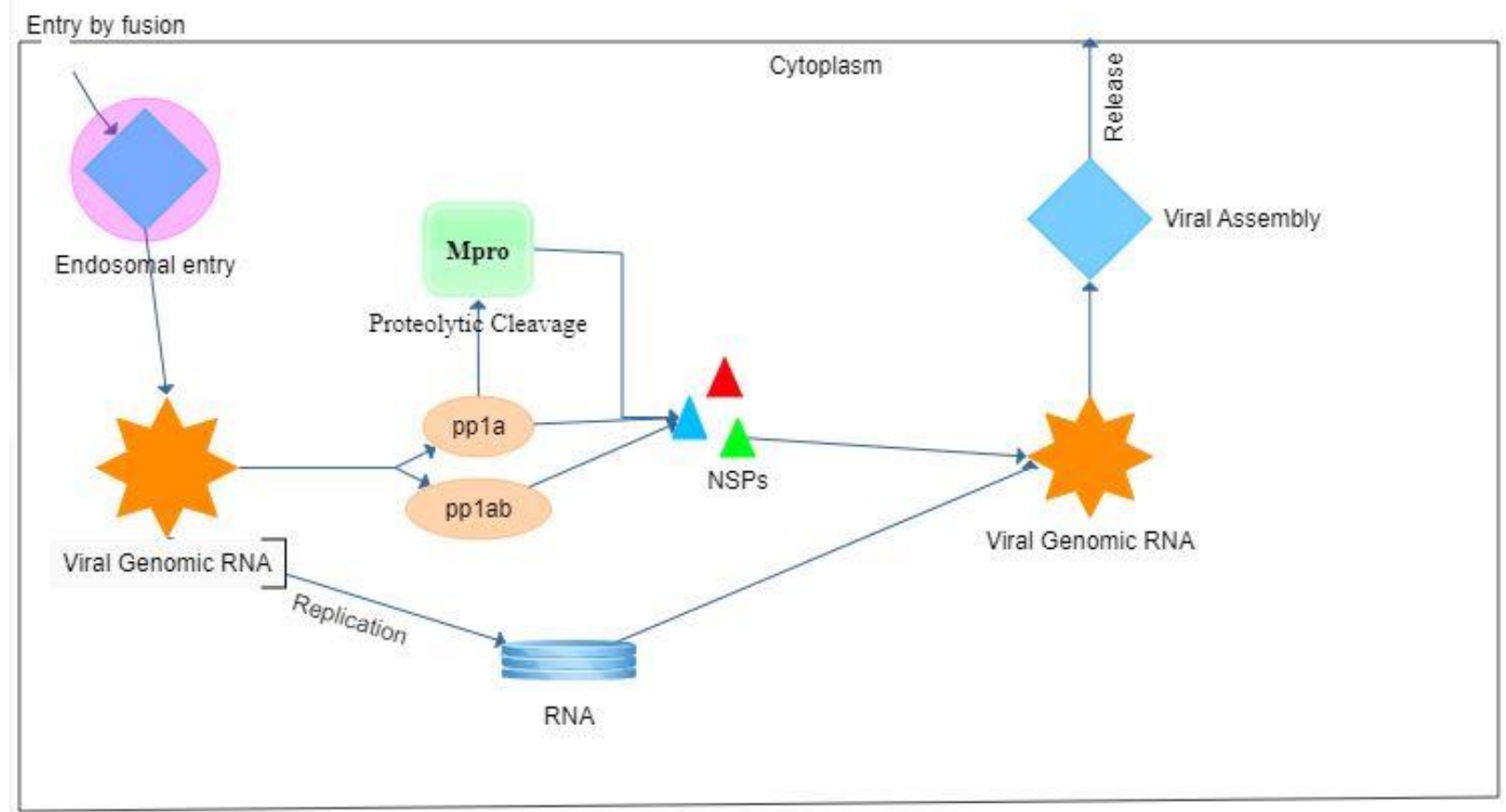

Figure 1: Mechanism of action of $\mathrm{M}^{\text {pro }}$ upon coronavirus entry

\section{Bioactive Compounds in Neem}

Traditionally agents from natural sources especially plants have been the main source of disease control. Different parts of neem, Azadirachta indica, have been used for thousands of years in Indian and African continents. The tree's Sanskrit name was 'arishtha', meaning 'reliever of sicknesses'. Neem flowers, leaves, seeds, bark etc. have a wide range of medicinal properties and are used to treat inflammation, infections, fever, skin diseases and dental disorders Neem constitutes a vast collection of biologically active compounds that are chemically diverse and structurally complex which are effective as antiviral antimicrobial, antimalarial and antibacterial. There are 140 active compounds isolated from different parts of the neem (Kumar et al.,2018). Neem leaf and its constituents exhibit antiviral, antifungal, antibacterial, immunomodulatory, antiinflammatory, antihyperglycemic, antiulcer, antimalarial, antioxidant, antimutagenic and anticarcinogenic properties. The beneficial effects of different parts of neem are attributed to its biologically active principal compound 'Azadirachtin'. A few other compounds include Nimbin, Nimbidin, Gedunin, Azadirone, Salanin, Cyclic tetrasulfide, Mahmoodin, Margolone and Catechin (Mohammad \& Forough, 2017). Neem leaves, a natural source of flavonoids, polyphenols, isoprenoids, sulphurous and polysaccharides, play an important role in scavenging the free radical and subsequently arresting disease pathogenesis.

Gedunin is a pentacyclic triterpenoid found in Azadirachta indica and Cedrela odorata. 
It is an antimalarial, an antineoplastic agent, a Hsp90 inhibitor and a plant metabolite. It is a limonoid, an acetate ester, an epoxide, an enone, a member of furans, a pentacyclic triterpenoid, an organic heteropentacyclic compound and a lactone (Jang et al.,2010).

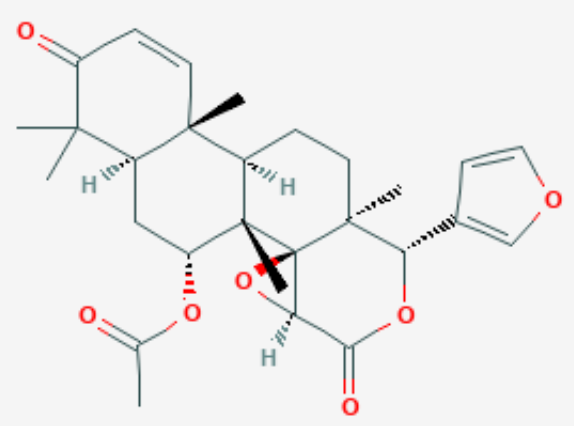

Figure 2: Molecular formula and structure of Gedunin $\underline{\mathrm{C}}_{28} \underline{\mathrm{H}}_{34} \underline{\mathrm{O}}_{7}$. Retrieved from PubChem.

IUPACName: [7-(furan-3-yl)-1,8,12,16,16-pentamethyl-5,15-dioxo-3,6-

dioxapentacyclo[9.8.0.02,4.02,8.012,17]nona dec-13-en-19-yl] acetate; Mol. Wt= $482.6 \mathrm{~g} / \mathrm{mol}$.

\section{Methodology}

The in silco study of docking was conducted using Auto dock tools 1.5.6. This software was chosen for the study as it had good accuracy. The crystal structure of the viralexclusive protein responsible for the replication of the virus was obtained from Protein Data bank (https://www.rcsb.org/structure/6LU7 PDB ID:6LU7).

The docking parameters are as follows: Lamarckian Genetic Algorithm and genetic algorithm (short/medium run). The
Macromolecule (6LU7) selected was docked with two major compounds present in Azadirachta indica: Gedunin and Nimbin separately. By employing this method, we can determine the binding energy and the conformations which have the least binding energy. This method is used as it elucidates the thermodynamic stability of the ligand bound to the protein.

\section{Results and discussion}

Table 1 shows the various conformations obtained from blind docking results using Autodock for Gedunin and Nimbin molecules as ligands docked with $\mathbf{M}^{\text {pro }}$ structure. 
Table 1: Binding energy, inhibition constant and intermolecular energies of various conformations of Gedunin and Nimbin arranged in decreasing order of preference

\begin{tabular}{|c|c|c|c|}
\hline Compound & $\begin{array}{c}\text { Binding } \\
\text { energy(kcal/mol) }\end{array}$ & Inhibition constant & $\begin{array}{c}\text { Intermolecular } \\
\text { energy }\end{array}$ \\
\hline \multicolumn{4}{|c|}{ Gedunin } \\
\hline Conformation 1 & -5.3 & 130.55 & -6.12 \\
\hline Conformation 2 & -5.67 & 69.68 & -6.49 \\
\hline Conformation 3 & -5.53 & 88.8 & -6.35 \\
\hline Conformation 4 & -6.74 & 11.37 & -7.57 \\
\hline Conformation 5 & -5.36 & 117.05 & -6.19 \\
\hline Conformation 6 & -6.14 & 31.84 & -6.96 \\
\hline Conformation 7 & -5.31 & 128.87 & -6.13 \\
\hline Conformation 8 & -5.22 & 149.11 & -6.04 \\
\hline Conformation 9 & -4.77 & 318.61 & -5.59 \\
\hline Conformation 10 & -6.88 & 9.04 & -7.7 \\
\hline \multicolumn{5}{|c|}{ Nimbin } \\
\hline Conformation 1 & -4.57 & 448.77 & -6.76 \\
\hline Conformation 2 & -4.16 & 890.95 & -6.36 \\
\hline Conformation 3 & -4 & 1.18 & -6.19 \\
\hline Conformation 4 & -3.35 & 3.53 & -5.54 \\
\hline Conformation 5 & -4.95 & 233.64 & -7.15 \\
\hline Conformation 6 & -5.35 & 120.19 & -7.54 \\
\hline Conformation 7 & -3.13 & 5.06 & -5.33 \\
\hline Conformation 8 & -5.12 & 177.66 & -7.31 \\
\hline Conformation 9 & -4.42 & 573.27 & -6.62 \\
\hline Conformation 10 & -3.82 & 1.58 & -6.02 \\
\hline
\end{tabular}

Neem is one of the key ingredients in ayurvedic treatment. It has proved to be an effective antiviral medication. It is now being used in ayurvedic treatments to cure covid. The results of in silico docking have shown that both Nimbin and Gedunin show good binding against $\mathrm{M}^{\text {pro }}$. Binding energy is a measure of the energy released upon the binding of a ligand to the active site of the protein target. This causes the overall energy of the complex to reduce. Lower binding energy indicates that the ligand has bound to 
the protein molecule spontaneously without the consumption of energy. The docking studies of the ligand-protein (6LU7 AND Gedunin) complex shows us that the binding energy is in the range -4.77 to -6.88 and that between 6LU7 and Nimbin complex is in the range between -3.13 to -5.35 .

Similarly, the inhibition constant values fall between 9.04 - 918.61 and 1.18 - 890.95 for Gedunin and Nimbin respectively. The inhibition constant describes the efficacy of a ligand against its target protein. A higher inhibition value is generally preferred for a ligand to be considered as a potential drug.
Intermolecular energy decreases in both Gedunin and Nimbin with the decrease in binding energy values ranging from -5.59 to -7.7 for Gedunin and 5.54 to -7.54 for Nimbin.

Based on the above results, considering binding energy, intermolecular energy and inhibition constant, it is observed that conformation 1 has the best binding scores and maximum efficacy for both Gedunin and Nimbin. The below figures show the binding of the molecules with the main protease enzyme, inhibiting its function.

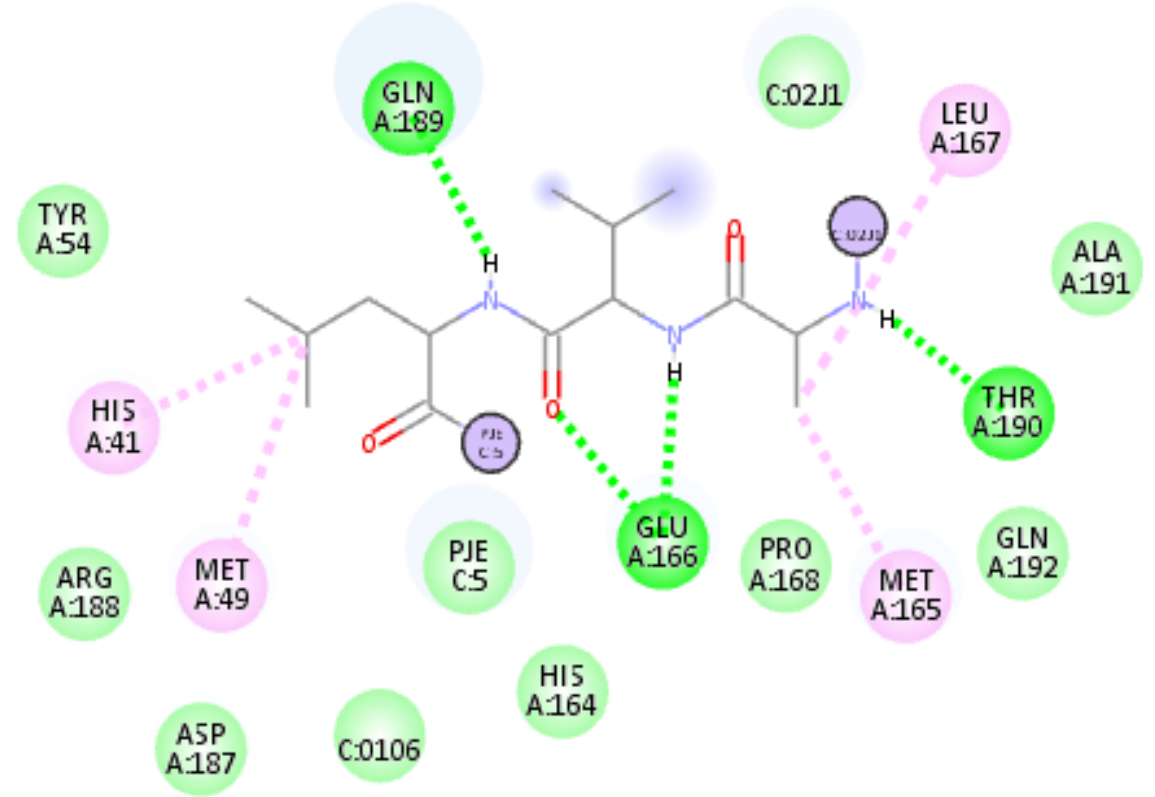

Interactions
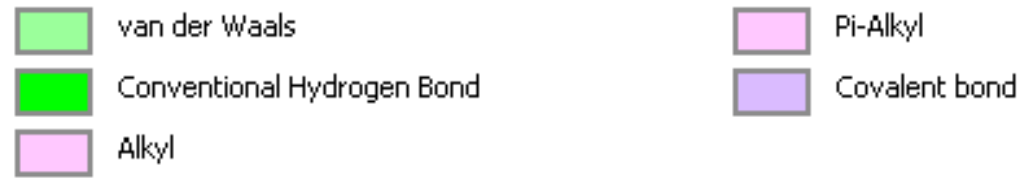

Fig 3 shows the interaction of Gedunin and Mpro 


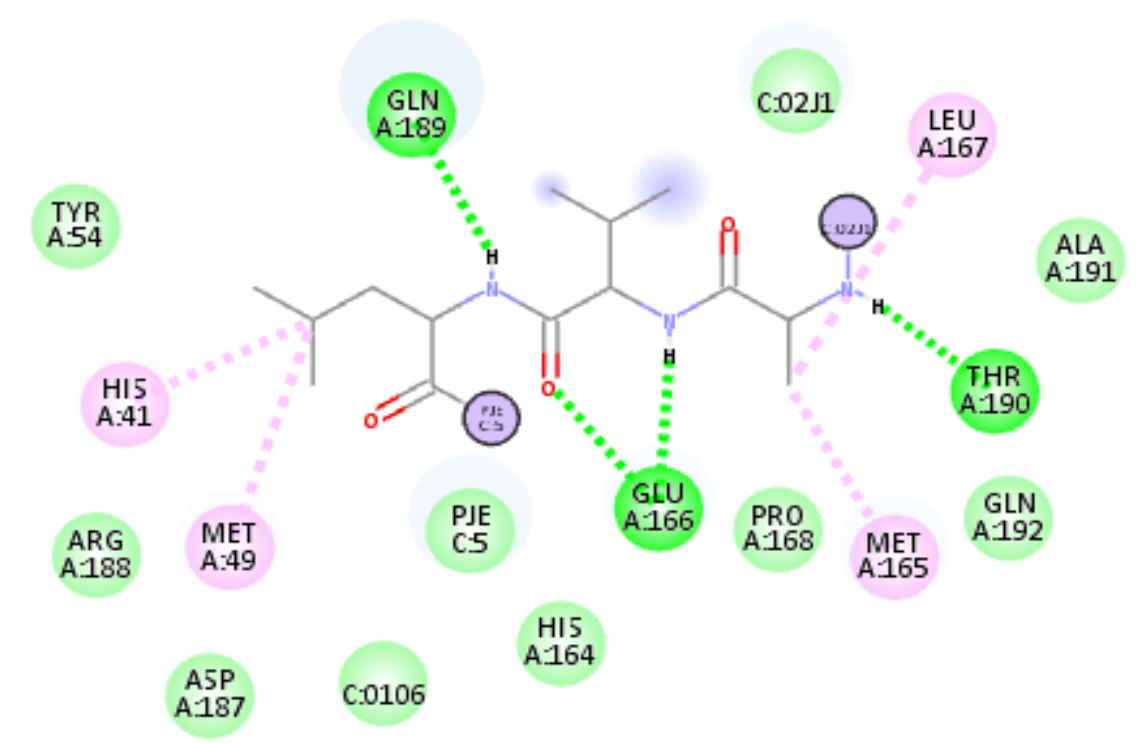

Interactions

van der Waals

Pi-Alkyl

Conventional Hydrogen Bond

Covalent bond

Fig 4 shows the interaction of Nimbin and Mpro

Despite the highly preferable values in silico, these ligands alone might not be able to yield the desired efficacy as that of raw and fresh neem leaves, which is found to cure mild to moderate covid-19 disease in individuals (Lim et al., 2021) and also it suppresses the replication of viruses significantly thus reducing the severity of the disease. We propose that the various other bioactive compounds present in the neem leaves act in conjunction to elicit the desired response. The paper names this property 'emergence' for the first time in biopharmaceuticals and drug design. Further research in the field

could better explain this phenomenon for more efficient drug designing.

\section{Conclusion}

This study elucidates how Gedunin and Nimbin bind to the target protein $\mathrm{M}^{\text {pro }}$ to inhibit its activity, thereby hindering viral replication. We also propose the property of emergence as the reason for the reduced efficacy of individual isolation and administration of the compounds. Further to molecular docking of the compounds, in vitro and clinical testing are required by the appropriate formulation of these compounds. We also suggest that further research is 
required to study the property of emergence in the field of therapeutics.

\section{Acknowledgement}

We thank Dr. Rajagopal Kamath for providing his support to conduct this study.

\section{References}

1. Anand, K., Palm, G. J., Mesters, J. R., Siddell, S. G., Ziebuhr, J., \& Hilgenfeld, R. (2002). The structure of coronavirus main proteinase reveals combination of a chymotrypsin fold with an extra alpha-helical domain. The EMBO journal, 21(13), 3213-3224. https://doi.org/10.1093/emboj/cdf327

2. Anand, K., Ziebuhr, J., Wadhwani, P., Mesters, J. R., \& Hilgenfeld, R. (2003). Coronavirus main proteinase (3CLpro) structure: basis for design of anti-SARS drugs. Science (New York, N.Y.), 300(5626), 1763-1767. https://doi.org/10.1126/science.1085 658

3. Chan JF, Yuan S, Kok KH et al. A familial cluster of pneumonia associated with the 2019 novel coronavirus indicating person-toperson transmission: a study of a family cluster. Lancet 2020. S01406736(20) 30154-9. 10.1016/S01406736(20)30154-9

4. Chen, N., Zhou, M., Dong, X., Qu, J., Gong, F., Han, Y., ... \& Zhang, L. (2020). Epidemiological and clinical characteristics of 99 cases of 2019 novel coronavirus pneumonia in
Wuhan, China: a descriptive study. The lancet, 395(10223), 507-513.

5. Grant, M. C., Geoghegan, L., Arbyn, M., Mohammed, Z., McGuinness, L., Clarke, E. L., \& Wade, R. G. (2020). The prevalence of symptoms in 24,410 adults infected by the novel coronavirus (SARS-CoV-2; COVID19): A systematic review and metaanalysis of 148 studies from 9 countries. PloS one, 15(6), e0234765.

6. Hilgenfeld, R. (2014), From SARS to MERS: crystallographic studies on coronaviral proteases enable antiviral drug design. FEBS J, 281: 40854096.

https://doi.org/10.1111/febs. 12936

7. Holshue ML, DeBolt C, First Lindquist $\mathrm{S}$ et al. Novel Coronavirus in the United States. $N$ Engl J Med 2019.

8. Jang, S. W., Liu, X., Chan, C. B., France, S. A., Sayeed, I., Tang, W., ... \& Ye, K. (2010). DeoxyGedunin, a natural product with potent neurotrophic activity in mice. PloS one, 5(7), e11528.

9. Jin, Z., Du, X., Xu, Y. et al. Structure of Mpro from SARS-CoV-2 and discovery of its inhibitors. Nature 582, 289-293 (2020). https://doi.org/10.1038/s41586-0202223-y

10. Kumar, R., Mehta, S., \& Pathak, S. R. (2018). Bioactive constituents of neem. In Synthesis of medicinal agents from plants (pp. 75-103). Elsevier.

11. Li Q, Guan X, Wu P et al. Early transmission dynamics in Wuhan, 
China, of novel coronavirus-infected pneumonia. N Engl J Med 2020. 10.1056/NEJMoa2001316

12. Lim XY, Teh BP and Tan TYC (2021) Medicinal Plants in COVID19: Potential and Limitations. Front. Pharmacol. 12:611408. doi: 10.3389/fphar.2021.611408

13. Mengist, H.M., Fan, X. \& Jin, T. Designing of improved drugs for COVID-19: Crystal structure of SARS-CoV-2 main protease Mpro. Sig Transduct Target Ther 5, 67 (2020). https://doi.org/10.1038/s41392-0200178-y

14. Mohammad Mehdi Sadeghian and Forough Mortazaienezhad, 2007. Investigation of Compounds from Azadirachta indica (Neem). Asian Journal of Plant Sciences, 6: 444445.

15. Richardson, P., Griffin, I., Tucker, C., Smith, D., Oechsle, O., Phelan, A., ... \& Stebbing, J. (2020). Baricitinib as potential treatment for 2019-nCoV acute respiratory disease. Lancet (London, England), 395(10223), e30.

16. Rottier PJM. The Coronaviridae. Siddell SG, editor. 115-137. 2013. Springer Science \& Business Media. (Available from: https://link.springer.com/content/pdf/ 10.1007\%2F978-1-4899-15313_6.pdf).

17. Tan, J., Verschueren, K. H., Anand, K., Shen, J., Yang, M., Xu, Y., Rao, Z., Bigalke, J., Heisen, B., Mesters, J. R., Chen, K., Shen, X., Jiang, H., \& Hilgenfeld, R. (2005). pH-dependent conformational flexibility of the SARS-CoV main proteinase (M(pro)) dimer: molecular dynamics simulations and multiple X-ray structure analyses. Journal of molecular biology, 354(1), 25-40. https://doi.org/10.1016/j.jmb.2005.0 9.012

18. Tyrrell DA, Bynoe ML. Cultivation of viruses from a high proportion of patients with colds. Lancet 1966: 1: 76-77. [PubMed] [Google Scholar]

19. Velavan, Thirumalaisamy $P$, and Christian G Meyer. "The COVID-19 epidemic." Tropical medicine \& international health: $T M \& I H$ vol. 25,3 (2020): 278-280. doi:10.1111/tmi.13383

20. Wang M, Cao R, Zhang L et al. Remdesivir and chloroquine effectively inhibit the recently emerged novel coronavirus (2019$\mathrm{nCoV})$ in vitro. Cell Res 2020. 10.1038/s41422-020-0282-0

21. Yang, J., Petitjean, S.J.L., Koehler, M. et al. Molecular interaction and inhibition of SARS-CoV-2 binding to the ACE2 receptor. Nat Commun 11, 4541

(2020). https://doi.org/10.1038/s41467-02018319-6

22. Zhang, L., Lin, D., Sun, X., Curth, U., Drosten, C., Sauerhering, L., Becker, S., Rox, K., \& Hilgenfeld, R. (2020). Crystal structure of SARS-CoV-2 main protease provides a basis for design of improved $\alpha$-ketoamide inhibitors. Science (New York, N.Y.), 368(6489),

409-412. 
https://doi.org/10.1126/science.abb3

405 